\title{
ILCEA
}

Revue de l'Institut des langues et cultures

d'Europe, Amérique, Afrique, Asie et Australie

14 | 2011

Traduction et Ergonomie

\section{La main et le clavier : histoire d'un malentendu}

Hand and Keyboard: the Story of a Misunderstanding

\section{Martine Pineau}

\section{OpenEdition \\ Journals}

Édition électronique

URL : https://journals.openedition.org/ilcea/1067

DOI : 10.4000/ilcea.1067

ISSN : 2101-0609

Éditeur

UGA Éditions/Université Grenoble Alpes

Édition imprimée

ISBN : 978-2-84310-203-5

ISSN : $1639-6073$

Référence électronique

Martine Pineau, «La main et le clavier : histoire d'un malentendu », ILCEA [En ligne], 14 | 2011, mis en ligne le 30 juin 2011, consulté le 21 septembre 2021. URL : http://journals.openedition.org/ilcea/1067 ; DOI : https://doi.org/10.4000/ilcea.1067

Ce document a été généré automatiquement le 21 septembre 2021

(C) ILCEA 


\title{
La main et le clavier : histoire d'un malentendu
}

\author{
Hand and Keyboard: the Story of a Misunderstanding
}

\author{
Martine Pineau
}

1 Les traducteurs passent une bonne partie de leur vie professionnelle à écrire, et comme tous les écrivants, ils ont une relation souvent conflictuelle avec leur instrument d'écriture. Déjà, au début du XVIII ${ }^{\mathrm{e}}$ siècle, Ramazini (1778, p.141-143) consacrait un chapitre aux métiers de l'écriture dans son étude des maladies professionnelles des différents corps de métiers, en décrivant notamment ce qu'on n'appelait pas encore des troubles musculo-squelettiques.

Quel que soit l'instrument utilisé, l'écriture est traditionnellement perçue comme une activité purement intellectuelle, détachée de toute contrainte matérielle et physique. Or écrire met en jeu le corps et renvoie aux techniques du corps telles qu'elles sont définies par Marcel Mauss: "les façons dont les hommes, société par société, d'une façon traditionnelle, savent se servir de leur corps » (Mauss, 1936, p. 5). Bien que les nouvelles technologies tendent à nier son importance, il faut bien reconnaître que le corps oppose une belle résistance. Nous ne pouvons pas faire autrement que de l'utiliser pour agir. Pour écrire aussi.

Dans le cas de l'écriture avec un clavier, la simplicité apparente de la manœuvre cache une opération complexe. De plus, le clavier lui-même se trouve à la convergence de pratiques très variées et omniprésentes dans le travail et les loisirs. Bien que très présent dans la vie privée et dans la vie professionnelle, c'est un outil qui semble résister à une appropriation intime par son utilisateur. Son usage relève ordinairement de deux modes radicalement différents. La première est une utilisation intuitive, spontanée, que l'on pourrait qualifier de "percussion monodactyle", la seconde est une utilisation plus sophistiquée, apprise, à savoir la dactylographie. Si le présent article est construit en grande partie à partir d'observations récoltées sur le terrain, il s'appuie aussi sur des lectures, des rencontres, des réflexions de l'auteure, qui est l'une des rares professeures de dactylographie exerçant en France. 
Dans cet exposé, nous examinerons quelques spécificités de l'écriture au clavier comme activité physique, avant d'aborder l'invisibilité du clavier : comment cela se traduit dans les représentations, quelles sont les causes de cette invisibilité et quelles en sont les conséquences pour la santé publique, l'organisation du travail, la productivité. Enfin, nous poserons quelques jalons pour réconcilier la main et le clavier, le corps et l'écriture. Puisse cet article amener les traducteurs à considérer avec plus de sérénité leur instrument de travail !

\section{Écrire : une activité physique}

5 Écrire n'est pas seulement une activité intellectuelle, c'est une activité physique à part entière qui mobilise le corps dans son ensemble, y compris jusqu'aux jambes. Bien que l'usage du clavier ne soit ordinairement pas perçu comme une activité physique à risques, un parallèle intéressant peut être établi entre l'écriture et le sport.

\section{Écrire : un sport comme un autre}

6 Tout d'abord, l'écriture est, comme un sport, constituée de mouvements qui ont entre eux une forte cohérence interne. Supprimer ou modifier un mouvement de la séquence, c'est prendre des risques : le risque de la faute disqualifiante ou celui de la blessure physique, comme en haltérophilie, par exemple.

7 Ensuite, l'écriture et le sport, en dépit de leur apparence de pratiques spontanées, reposant sur des gestes maîtrisés de façon automatique, sont au contraire des activités physiques artificielles et culturelles. Rien n'est plus artificiel que la natation par exemple. Écrire et nager (ou n'importe quel autre sport) sont des pratiques apprises, élaborées, sophistiquées. Dans les deux cas, il s'agit de gestes créés par l'homme, qui n'ont rien à voir avec un geste "naturel », comme par exemple le saut en hauteur en position dorsale, inventé par Fosbury en 1968. Écrire aujourd'hui, c'est intégrer un long apprentissage et aussi une longue histoire de tâtonnements pédagogiques.

8 Troisième et dernier point : écrire et pratiquer un sport sont des activités qui mettent en œuvre chacune un ensemble technique particulier au sens donné par Gilbert Simondon (2001, p. 61) et une des pièces de cet ensemble technique est le corps humain. Sauf peutêtre lorsque l'on écrit avec le pouce du pied sur le sable de la plage, écrire nécessite non seulement l'emploi d'une partie du corps mais aussi d'un instrument précis et adapté au support et d'un aménagement de l'environnement, tout comme le cyclisme ou le ski mettent en œuvre des instruments complexes et nécessitent des aménagements spécifiques de l'environnement.

9 La question qui vient à l'esprit à propos de cet ensemble technique corps + machine + environnement est la suivante : est-ce le mouvement qui crée la machine? ou la machine qui crée le mouvement? Si l'on veut bien considérer que les mouvements de l'écriture (crayon ou clavier), tout comme la plupart des mouvements sportifs, sont aberrants au regard de notre capacité anatomique naturelle, on comprend alors que c'est plutôt la machine, ou l'instrument, qui nous a fait inventer les mouvements nécessaires à la mise en acte de notre imagination créatrice. C'est vrai pour le cyclisme (pédaler : voilà bien un mouvement circulaire sans contact avec le sol très récent dans l'histoire humaine) ; c'est vrai aussi pour l'écriture, a fortiori pour l'écriture au clavier. 


\section{Un outil figé, d'une extraordinaire pérennité}

10 L'ensemble technique utilisé pour écrire au clavier repose sur un outil figé, car le clavier tel qu'il est actuellement installé sur nos bureaux a fort peu évolué depuis 1880, en dépit de l'arrivée de l'informatique.

$11 \mathrm{Au}$ tout début, les caractères sont portés par des tiges métalliques, elles-mêmes actionnées par les touches du clavier. La contrainte mécanique est très forte puisqu'il faut éviter autant que possible que les tiges ne s'emmêlent et ne risquent de se tordre ; pour remédier à cet inconvénient, on associe alors deux solutions techniques. Premièrement, on dispose les touches en quinconce pour donner plus d'espace aux tiges d'une rangée de touches par rapport à la rangée immédiatement voisine au-dessus ou au-dessous. Deuxièmement, on intercale les caractères sur les touches de telle sorte que les caractères les plus fréquemment utilisés voisinent avec des caractères moins utilisés. Et comme, jusque vers 1890 , les dactylographes ne voient pas ce qu'ils écrivent, les touches les plus fréquemment utilisées, dont toutes les voyelles, sont disposées en haut du clavier pour qu'elles soient visibles, placées au-dessus des mains. Ainsi naquit QWERTY, suivi peu après par les grandes variantes régionales que sont AZERTY et QWERTZ.

12 Au centre du clavier, les caractères sont regroupés dans un pavé alphanumérique ; puis on ajoute une touche modificatrice qui permet de mettre les caractères en majuscule, ainsi qu'une touche de fonction (si on veut bien accepter cet anachronisme...), la touche de retour arrière qui se présente d'ailleurs à l'époque sous forme d'un levier latéral à la machine ${ }^{1}$.

13 Le clavier s'alourdit au fil des années, notamment avec l'arrivée de la microinformatique; les touches de fonction se multiplient, les touches spécifiques des différents constructeurs apparaissent, un pavé numérique séparé est ajouté ainsi qu'un pavé directionnel pour se déplacer plus commodément dans les documents en cours de travail. Tous ces éléments sont dispersés autour du pavé alphanumérique, sans grand souci d'accessibilité par les doigts quant à la symétrie ou à la distance. Autour d'un pavé alphanumérique déjà fort peu fait pour la main humaine... faut-il le rappeler.

14 Le résultat de tous ces ajouts conduit à des distorsions des mains, à des mouvements d'extension beaucoup trop fréquents, notamment vers le haut du clavier, à un sousemploi des pouces. Il faudrait y ajouter la grande dissymétrie induite par le clavier, puisque sur nos claviers ordinaires de type azerty, la disposition des touches favorise la main droite alors que les caractères les plus utilisés en français sont placés à gauche du clavier: une estimation empirique ${ }^{2}$ montre en effet qu'environ $60 \%$ de la frappe d'un texte en français se fait sur la partie gauche du clavier de type azerty. Pour poursuivre avec la métaphore sportive, c'est un peu comme si on utilisait des draisiennes pour le Tour de France.

Une fois ce constat établi, la pérennité du clavier inadapté que nous connaissons peut sembler une véritable énigme, qui pourrait s'expliquer en partie par l'inertie des fabricants et le figement de l'usage social (Jacomy, 2001).

$16 \mathrm{Au}$ tout début de sa carrière, le clavier était utilisé exclusivement en percussion monodactyle (comme le font la plupart des utilisateurs en ce début de xxI ${ }^{e}$ siècle), c'est-àdire avec souvent un seul doigt par main, l'index de préférence, éventuellement assisté par le majeur. Dans cette configuration gestuelle, le pointage exact du doigt se fait sous le 
contrôle des yeux qui, d'une part, regardent le clavier pour choisir la bonne touche et, d'autre part, vérifient sur le support d'écriture la conformité du résultat au souhait du percuteur. Au cours des années 1880, Mc Guring, champion de dactylographie, crée un geste spécialement adapté à la machine en utilisant ses dix doigts et surtout en affranchissant totalement l'écriture au clavier de la vision. La dactylographie qu'il crée ainsi repose uniquement sur le premier de nos sens: le toucher ${ }^{3}$. Améliorer la performance en créant un geste totalement nouveau, c'est aussi ce qu'avait fait Johnny Weissmuller en créant le crawl.

On peut faire un parallèle entre l'écriture au clavier et l'écriture au crayon: tenir un crayon se fait spontanément à pleine poigne, comme le font les petits enfants. Mais écrire en tenant le crayon de cette façon est extrêmement exigeant pour tout le haut du corps puisqu'il est nécessaire d'activer tous les muscles du membre supérieur et ceux d'une bonne partie du dos. Le geste le plus économique est en fait le plus artificiel, qui consiste à ne tenir et à n'actionner le crayon qu'avec les doigts, spécialement entre le pouce, l'index et le majeur. Le poignet est alors moins sollicité, le bras encore moins et le dos presque pas.

Écrire en percussion monodactyle sur un clavier, c'est reproduire le schéma de l'écriture au crayon tenu à pleine poigne en faisant travailler tout le haut du corps. Les victimes privilégiées de cette contrainte gestuelle sont les vertèbres cervicales, mais aussi les épaules et les poignets. Sans oublier les yeux car pour écrire une ligne sur un document de format A4, avec des caractères de chasse moyenne en corps 9 , le percuteur moyen regarde alternativement clavier et écran en moyenne 3 à 5 fois par ligne ${ }^{4}$. Les yeux sont sévèrement affectés par ce va-et-vient qui les asservit au couple clavier/écran : tous les ophtalmologues, médecins du travail, décrivent des phénomènes de fatigue visuelle, de syndrome d'œil sec, provoqués par une accommodation répétitive à une surface sombre (le clavier) qu'il faut déchiffrer, puis à une surface lumineuse (l'écran) qu'il faut contrôler, et en plus à une surface réfléchissante si le percuteur utilise encore du papier à côté de son clavier. L'œil surfocalise, "myopise» comme disent les médecins du travail. Au contraire, l'écriture dactylographique libère complètement la vision de cette fonction de contrôle et de coordination gestuelle pour restituer à l'œil son activité de lecture et sa capacité à bouger librement, ce qui est agréable en réunion, par exemple. L'écriture au clavier devient ainsi bien plus confortable que l'écriture au crayon.

\section{La preuve par les chiffres}

Deux expressions populaires évoquent clairement les aspects physiques de l'écriture au clavier : le poids des mots et la frappe au kilomètre.

Un traducteur disait un jour, en décrivant ses maux d'épaules : «Je ne fais pourtant pas de maçonnerie... » S'était-il seulement demandé quelle force est nécessaire pour activer les touches du clavier? On considère généralement qu'écrire 1000 mots en français nécessite en moyenne 5500 caractères $^{5}$ (en réalité davantage car certains caractères nécessitent deux frappes). La force idéale pour activer une touche correspondrait à $40 \mathrm{~g}$, soit $220 \mathrm{~g}$ par mot, et donc $220 \mathrm{~kg}$ pour 1000 mots. Dans la mesure où la plupart des traducteurs produisent en moyenne 2500 mots par jour, cela représenterait $550 \mathrm{~kg}$. Comme la force réelle nécessaire pour activer les touches de nos claviers excède très souvent les $50 \mathrm{~g}^{6}$, voire les $60 \mathrm{~g}$, et que le traducteur écrit souvent plus en une journée, ce dernier est souvent plus proche de la tonne quotidienne... 
21 Puisqu'activer les touches demande un effort, pourquoi ne pas choisir de frapper sur une surface plane, comme ces claviers virtuels qui sont projetés sur la surface dure du bureau? Mais l'absence de retour tactile complique sérieusement la tâche: le choc est alors brutal pour les doigts et le geste est totalement asservi au regard puisque l'on ne peut aucunement faire appel au toucher, ce qui contraint les utilisateurs de ce type de clavier à la percussion monodactyle, y compris ceux qui ont une compétence dactylographique.

La frappe au kilomètre est la deuxième expression qui vient assez spontanément à l'esprit lorsqu'on parle de dactylographie, et qui renvoie typiquement aux dactylos d'antan... Il est intéressant de donner un autre éclairage à cette expression. Pour un dactylographe qui écrit avec un clavier classique de type AZERTY, la distance parcourue par les doigts (toujours pour 1000 mots de 5,5 caractères) est d'environ ${ }^{7} 174 \mathrm{~m}$. Pour les percuteurs monodactyles, ce chiffre peut être multiplié par 2, voire par 3... puisque les mouvements des doigts ne sont pas définis à partir d'une position de repos. En particulier, le ou les deux doigts utilisés de la main droite font des trajectoires étonnantes et démesurées en couvrant très souvent les $3 / 4$ du clavier, ce qui rappelle tout à fait l'allure des cyclistes grimpant les cols pyrénéens avec de vastes mouvements d'épaules...

Par ailleurs, il convient de remarquer qu'écrire est une activité mentale qui supporte difficilement d'être entravée par les activités que sont : épeler, copier ou parler.

L'épellation est présente chez les percuteurs mono-digitaux et donne souvent lieu à une articulation muette du son par la bouche, ce qui, chez des adultes lettrés, ne se produit jamais lorsqu'ils écrivent avec un crayon. Beaucoup trop de personnes préfèrent encore rédiger d'abord avec papier et crayon (c'est-à-dire : penser) avant d'écrire au clavier (c'est-à-dire: exécuter). L'épellation conduit ainsi à dissocier l'activité purement intellectuelle (car soutenue par l'écriture au crayon complètement intégrée comme automatisme par le sujet) de l'activité physique de saisie au clavier qui n'est pas une activité réflexe pour ces personnes. La copie, dans le contexte professionnel actuel, se présente surtout sous la forme de l'auto-copie, encore assez largement pratiquée, y compris par les traducteurs; elle ajoute de la contrainte par la lecture qu'elle nécessite, voire le déchiffrement s'il s'agit d'une écriture manuscrite, y compris la sienne propre... Enfin, la dictée (écrire en parlant) fait un grand retour grâce aux logiciels de dictée ${ }^{8}$ qui ont avantageusement remplacé les anciens dictaphones. D'autres problèmes linguistiques et ergonomiques apparaissent alors, induits par l'effort d'exprimer oralement en un seul jet un discours destiné à l'écrit et par la fatigue des cordes vocales que connaissent les personnes non formées au travail avec la voix.

Épeler, copier et parler, ces activités parasites sont des sources de surcharge mentale dont le travail intellectuel n'a pas besoin et qui donnent à comprendre toute la distinction que les ergonomes établissent entre le travail prescrit et le travail réel (Niezborola, 2007). Cet écart est en soi une source de stress important.

\section{Une activité invisible avec un outil invisible}

\section{Un constat}

Écrire au clavier est une activité invisible dans les arts, en particulier dans le cinéma français et européen. En général, les acteurs français et européens tapent en monodactyle 
ou font semblant. Par exemple, dans le récent film roumain intitulé Policier: adjectif, réalisé par Corneliu Porumboiu, une séquence montre le doigté de l'actrice filmé de très près, mais écrire en roumain sans utiliser de « $\mathrm{i} »$ ni de « $u$ » semblera à d'aucuns pour le moins hasardeux.

Toutefois, dans ces tentatives cinématographiques de reconstitution des gestes dactylographiques, il est intéressant d'observer que les acteurs recherchent spontanément le confort en plaçant leurs mains plutôt sur le bas du clavier et en activant leurs mains de façon plutôt parallèle. En revanche, la dactylographie étant enseignée dans le cursus scolaire américain (secondaire et/ou supérieur), beaucoup d'acteurs américains dactylographient réellement au cinéma, y compris dans les séries télévisuelles : on y voit des doigtés bien coordonnés qui témoignent d'une pratique réelle, pas seulement d'une reconstitution pour les besoins du tournage.

On retrouve ce clivage européen/américain dans la littérature. Le seul genre littéraire dans lequel le clavier est pratiqué des deux côtés de l'Atlantique est la littérature policière; certains auteurs français lui donnent même une place de choix (Simenon, Vargas). Mais le clavier et son usage sont généralement ignorés des autres genres littéraires en français. En témoignent, presque d'une façon caricaturale, les propos d'Amélie Nothomb qui a affirmé ne pas répondre aux courriels : « D'abord parce que je ne possède pas d'ordinateur. La graphie est pour moi un acte hautement physique. Ensuite, pour avoir lu certains mails que l'on m'avait transmis, j'ai observé qu'ils étaient moins intéressants et montraient moins de tenue que les lettres ${ }^{9}$.» On pourra apprécier tout ce qu'il y a de posture dans ce refus d'utiliser un outil technologique contemporain - alors que $\mathrm{M}^{\text {lle }}$ Nothomb utilise très certainement la voiture, le lave-vaisselle... qui pourtant nous privent des gestes essentiels que sont marcher avec nos pieds ou travailler avec nos mains. Les écrivains américains écrivent au clavier, depuis longtemps et dans tous les styles de littérature. Les témoignages sont nombreux, et on en retiendra le beau texte de Paul Auster (Auster, 2003) qui atteste d'une quasi-amitié pour son instrument d'écriture. Enfin, peu de claviers figurent sur la scène théâtrale, dans la peinture ou dans la sculpture : même le sculpteur César n'a pas jugé le clavier digne de compression.

Bien que pratiquée dans toutes les entreprises, l'écriture au clavier est également une activité invisible dans le monde de l'entreprise : peu de cadres des ressources humaines savent comment les salariés utilisent leur clavier : «ils se débrouillent » est la phrase la plus souvent entendue. Les responsables ne savent pas combien de mots chaque salarié écrit par jour, combien d'heures il passe sur son clavier, et ils ne s'intéressent pas au coût associé à cette activité. Le clavier est d'ailleurs absent du Document unique (DU) que les entreprises doivent établir pour l'évaluation des risques du travail. Les Comités d'hygiène, de sécurité et des conditions de travail (CHSCT) ignorent également son existence.

De plus, le clavier est un outil mal acheté, qui ne bénéficie pas de critères d'achat, à l'exception du prix et de quelques tendances « mode » comme le coloris (noir) ou le rétroéclairage. C'est d'ailleurs un outil mal vendu, tant dans les grandes surfaces que dans les magasins spécialisés où il est le plus souvent relégué au rayon des accessoires de l'informatique, en bas des rayonnages, en fin de console; il est rarissime de trouver un vendeur ayant une bonne connaissance du clavier et de son usage. Même pour des organismes tels que UFC Que choisir ou Soixante millions de consommateurs, le clavier d'ordinateur est inexistant, alors que le clavier de téléphone portable nourrit des 
rubriques entières. Encore ce dernier ne fait-il l'objet d'enquête que par rapport à sa durée de vie, et non pas à son usage ni à son utilisabilité.

31 L'invisibilité du clavier est ancienne et profondément incrustée dans nos représentations. À l'époque de l'arrivée massive de la micro-informatique dans les entreprises, le décret du 14 mai $1991^{10}$ relatif à la prévention des risques liés au travail sur des équipements comportant des écrans de visualisation [...] consacrait un seul article au clavier, sur les quinze articles le composant. L'invisibilité du clavier est entérinée de fait par ce décret consacré essentiellement au nouveau signe de modernité: l'écran. Le clavier, difficilement évitable, est tout juste toléré dans l'appareil règlementaire français. Il convient donc de s'interroger sur les causes de cette invisibilité, constatée aussi bien dans les arts, les entreprises et les commerces que dans l'appareil règlementaire français.

\section{Les causes}

Les travaux historiques de Delphine Gardey (1999) donnent un éclairage intéressant que l'on peut résumer en deux mots : taylorisation et féminisation. En effet, l'organisation du travail initiée par Taylor a également transformé les pratiques professionnelles du tertiaire. L'écriture, qui a toujours été un goulet d'étranglement dans la transmission de l'information, est l'une des premières tâches concernées. Avec une organisation tayloriste du travail, apparaît, à partir des années 1910-1915, une écriture segmentée entre de nombreuses personnes, impliquant jusqu'à 5 ou 6 intervenants : celui qui dicte (donc qui pense), celui ou celle qui rédige, celle qui recopie, celui ou celle qui relit, celui qui signe (qui a vraiment le pouvoir et qui selon les entreprises était ou non celui qui avait dicté) ${ }^{11}$. L'organisation suit le modèle industriel, avec un pool de plusieurs dizaines d'employées, les fameuses " dactylos ${ }^{12}$ » qui travaillent souvent en blouse pour ne pas se tacher avec l'encre des rubans encreurs, dans un lieu terriblement bruyant très proche de l'atelier d'usine. Le travail est souvent payé au rendement, ce qui le rapproche du travail de l'OS (ouvrier spécialisé) et ce type d'organisation perdure jusqu'aux années 1970-1980.

De 1880 à 1910, l'écriture dactylographique est une tâche parmi d'autres, intégrée dans la pratique professionnelle ordinaire des employés du tertiaire. Et de ce fait, avant 1910, la dactylographie est majoritairement une pratique masculine $e^{13}$, puisque la plupart des employés de bureau sont des hommes.

34 À partir de 1910, et surtout après 1918, la dactylographie devient un métier exclusivement féminin, souvent perçu comme une activité temporaire en attendant le mariage, et sans évolution de carrière possible. Du fait de sa féminisation, la compétence est perçue de plus en plus comme reposant uniquement sur une aptitude naturelle, bien que l'apprentissage soit exigeant, notamment pour l'acquisition de la vitesse et d'un bon niveau de correction orthographique. Combien de femmes ayant exercé le secrétariat se sont entendu dire qu'elles étaient douées " pour ça », le «ça » désignant un seul de tous leurs instruments de travail : le clavier, et pas le téléphone qui était d'emblée mixte, ni aucun autre instrument de travail de bureau même si elles étaient souvent les seules à les utiliser, comme la Gestetner ou la photocopieuse plus récemment.

C'est en devenant une pratique très précisément quantifiable, du fait de sa mécanisation, que l'écriture dactylographique a perdu le prestige d'activité intellectuelle. Ce point est important pour expliquer, spécialement en France, le dédain pour l'écriture au clavier. Dans les représentations socio-culturelles des Français, l'opposition traditionnelle entre profession intellectuelle et profession manuelle est très fortement ancrée. D'ailleurs dans 
notre système éducatif, la main est totalement négligée et n'est formée à aucun savoirfaire, si ce n'est dans les filières techniques courtes.

L'écriture dactylographique est donc descendue bien bas dans l'échelle du travail tertiaire. Elle est devenue une pratique honteuse et disqualifiante ; cela explique la forte réticence qu'éprouvent un grand nombre de femmes cadres dans les entreprises actuelles pour apprendre à dactylographier, encore de nos jours. Nous citerons pour l'anecdote la réaction d'une ingénieure, à qui cette formation était suggérée : «Ah non! Ils vont aussi me demander de taper leurs textes!»

\section{Les conséquences}

Les conséquences de cette invisibilité sont nombreuses. Tout d'abord la formation dactylographique, qui a longtemps été réservée exclusivement à la filière " secrétariat ", a été totalement abandonnée par l'Éducation nationale depuis une douzaine d'années et remplacée par la formation aux fonctionnalités bureautiques de base. Fort bien, mais une fois que l'on comprend comment fonctionne la machine, sait-on s'en servir efficacement? La dactylographie ne fait pas l'objet non plus de validation certificative de type PCIE $^{14}$.

De plus, pour ce qui concerne la plupart des méthodes de formation (en présentiel ou par le biais de divers tutoriels numériques), on observe que la pratique pédagogique est inchangée depuis 1910, basée uniquement sur la copie. Il n'est tenu compte ni des apports de l'informatique, ni des évolutions de l'organisation du travail, ni des évolutions de la pédagogie. La comparaison est intéressante avec l'apprentissage de la pratique sportive : plus personne n'enseignerait la natation comme en 1910, à plat ventre sur le bord de la piscine. Le récent rapport "Réussir l'école numérique " laisse peut-être entrevoir une ouverture en prévoyant « un apprentissage ludique du clavier » en maternelle et au début du primaire (Fourgous, 2010, p. 213), bien que l'on puisse s'interroger sur la valeur d'un tel enseignement destiné à des enfants pas encore complètement latéralisés et ne maîtrisant pas la lecture.

Les méfaits de la percussion monodactyle (ou de l'usage nécessairement improvisé du clavier) par la quasi-totalité de la population sont de trois ordres : les troubles musculosquelettiques (TMS), l'accessoirisation du poste de travail et les pertes de productivité.

Si les médecins du travail attribuent des causes multifactorielles au déclenchement des TMS, tous reconnaissent que la répétition de mouvements contraints, même de faible intensité, mal articulés entre eux et superflus, en sont les facteurs dominants. Ces troubles musculo-squelettiques diffus affectent les tissus mous péri-articulaires (muscles, tendons, nerfs) et se traduisent par des picotements, des fourmillements, des douleurs sourdes continues, y compris pendant le sommeil, ou bien par des douleurs vives au moment de l'effort, des engourdissements jusqu'à des difficultés à saisir des objets. Les plus fréquents des TMS liés à l'usage du clavier sont le syndrome du canal carpien pour le poignet et l'épicondylite pour le coude ; mais les épaules sont aussi touchées, ainsi que les vertèbres cervicales. Le risque est maintenant bien identifié, y compris par les fabricants, dont certains ont la bonté d'imprimer une mise en garde sur la notice accompagnant le clavier, ou directement sur l'envers du clavier ${ }^{15}$.

41 L'ordre habituel de riposte face à ce genre de problème est le suivant : d'abord un recours à l'automédication avec anti-inflammatoire, puis une consultation auprès du médecin de famille qui ne fait pas toujours le lien avec le travail effectué et qui recommande des 
séances auprès d'un kinésithérapeute, mais aussi assez souvent l'arrêt du sport, de la musique, de toute activité de loisir affectée par un ressenti douloureux; sans compter le recours à des médecines douces plus ou moins aléatoires. Les médecins du travail déplorent la sous-déclaration par les salariés, surtout si le médecin est lui-même salarié de l'entreprise. Au final, le remède est chirurgical. Le coût moyen d'une opération du canal carpien est de $9000 €$ en 2009. Avant d'en arriver là, il y aura eu des arrêts de travail à répétition. La grande question actuelle est donc de passer de la réparation du dommage corporel à la prévention, entre autres par le recours à l'expertise des ergonomes.

Pour pallier l'inertie des utilisateurs et les insuffisances du clavier, les accessoires se multiplient ${ }^{16}$. On trouve ainsi des soutiens pour diverses parties du corps comme si nous étions menacés d'écroulement, tel celui qui est abusivement nommé appuie-poignet ${ }^{17}$, l'appuie-coude, etc. Ces accessoires sont certes très utiles pour soulager momentanément une personne qui souffre mais ne sauraient apporter une solution définitive ni tenir lieu d'aménagement du poste de travail.

« La productivité du clavier [...] n'a que médiocrement progressé dans ces deux dernières décennies alors que, dans un même temps, tous les autres composants du microordinateur [...] ont fait un bond spectaculaire", peut-on lire dans un rapport gouvernemental qui souligne ce goulot d'étranglement des TIC qu'est la saisie (Trégouët, 1998). Un des critères traditionnellement retenus pour mesurer la productivité au clavier est la vitesse de frappe. Encore convient-il de savoir ce que l'on mesure lorsqu'on parle de vitesse. Cette vitesse est ordinairement mesurée en situation de copie et ne peut valoir que sur une durée probante, comme en situation du championnat Intersténo ${ }^{18}$ dont l'épreuve de dactylographie dure trente minutes. Or si, comme toute épreuve sportive, un concours de dactylographie requiert des qualités athlétiques et esthétiques propres, la vitesse atteinte par les champions, mesurée en frappes par minute, ne saurait servir à déterminer le standard des pratiquants ordinaires.

En dehors des chronométrages établis en compétition, la perception de la vitesse est d'ailleurs assez largement subjective. On entend souvent dire «je tape vite », phrase qui témoigne de la confusion bien établie entre bouger vite et écrire vite. Quand le chronomètre impitoyable se met en marche, taper vite revient dans le meilleur des cas à écrire environ 24 mots par minute ${ }^{19}$, sur une séquence de 3 à 4 minutes, (au moyen d'une gesticulation effrénée) c'est-à-dire très loin des 30 minutes de compétition ${ }^{20}$... Dans la vie professionnelle ordinaire, plutôt que de vitesse brute, il semble plus convenable de remédier à un taux d'erreurs de frappe élevé, de privilégier la fluidité de l'écriture, sans épellation, qui donne à l'écrivant la possibilité de moduler le rythme en accélérant ou en ralentissant, pour suivre le fil de la pensée. Le gain de productivité se fera grâce à la rédaction en direct et à la suppression de la recopie. On parviendra ainsi à diminuer une grande part de stress, à réduire les congés maladie pour cause de TMS et aussi, de façon plus inattendue, à modérer la consommation de papier.

\section{Réconcilier la main et le clavier}

En l'état actuel des choses, il y a plusieurs voies pour réconcilier la main et le clavier, et la première est une formation adéquate à l'écriture mécanisée, tout comme on apprend à conduire ou à utiliser d'autres machines. Ce point ressort assez nettement de l'ensemble de l'exposé ci-dessus pour qu'on n'y revienne pas. D'autres axes de travail sont intéressants; ils offrent de plus l'avantage de ne pas être exclusifs l'un de l'autre. $\mathrm{Au}$ 
contraire, utilisés de façon conjointe, ils ont plutôt tendance à multiplier leurs effets bénéfiques.

\section{Adapter l'humain au travail}

Prise dans son acception brute, l'approche "gestes et posture", qui a été largement développée par les ergonomes, ressemble à première vue à un catalogue de prescriptions coercitives du type «tiens-toi droit, fais ceci, ne fais pas cela». Le profane peut difficilement la mettre en œuvre seul: elle demande l'intervention compétente d'un ergonome.

Une autre voie intéressante est d'agir sur l'organisation du travail, et tout spécialement son rythme. Les TMS sont provoqués, comme l'indique nettement l'anglais RSI (repetitive strain injury ${ }^{21}$ ) par la répétition des mouvements. Savoir détendre le corps par de brefs exercices qui rompent la contrainte peut apporter un grand bien-être. C'est la démarche des micro-pauses, brefs instants de détente de quelques dizaines de secondes consacrés à des étirements, à des automassages, à des exercices respiratoires ainsi qu'à quelques exercices de relaxation visuelle. Pour le travail d'écriture, on peut avec un certain profit utiliser un logiciel approprié2 ${ }^{22}$. D'une manière générale, les Nord-Américains, et spécialement les Canadiens, sont très en avance pour cette offre encore peu développée en France. Le reproche que l'on peut faire à ces logiciels est qu'ils sont trop intrusifs, un peu comme un réveille-matin opiniâtre. Le mieux est peut-être d'apprendre à écouter son ressenti corporel, à faire des exercices de relaxation au moment opportun.

\section{Adapter l'outil de travail à l'humain}

Les règles d'aménagement du poste de travail telles qu'elles sont définies, par exemple, par la brochure de l'INRS ${ }^{23}$ qui décrit très précisément les angles des bras contribuent également au confort de travail ${ }^{24}$. Mais comment optimiser le mouvement? Ces règles requièrent impérativement la médiation d'un ergonome pour être utilisées de façon pertinente. Dans les grandes entreprises, les postes de travail sont ordinairement aménagés selon un minimum de règles ergonomiques (mais pas toujours avec un ergonome). Mais cela ne suffit pas pour qu'ils soient bien utilisés ou suffisamment adaptés aux personnes qui les utilisent. Quant aux travailleurs indépendants, auto-entrepreneurs ou salariés de TPE (cas assez fréquent des traducteurs), ils sont ordinairement assez mal lotis et utilisent le plus souvent du mobilier inadapté. Or, même dans ce cas, il n'est pas inconsidéré de faire appel à un ergonome pour installer un bureau et pour choisir le mobilier professionnel. Cela peut éviter bien des tâtonnements, des dépenses inutiles et au final des douleurs superflues.

En particulier pour le siège, l'évolution générale est à la mobilité et à l'adaptabilité. Alors que pendant longtemps, le siège était fixe, à la même hauteur pour tous et pour toutes les occupations, la tendance actuelle est de privilégier le mouvement et la liberté du corps (Jouvin, 2006). Les sièges sont ainsi passés de l'assise fixe avec accoudoirs et dossier droit pour se « tenir droit » à une assise mobile plus souple. Acheter un siège de bureau mérite un essayage préalable, bien que cela soit parfois difficile car tous les choix ne sont pas toujours disponibles. Le mieux est encore d'aller visiter les salons consacrés à la prévention comme Préventica ${ }^{25}$, Ergonoma ${ }^{26}$ ou encore les salons consacrés à l'équipement de bureau ${ }^{27}$. 
50 Adapter le clavier à la langue écrite est une autre voie d'amélioration du confort de travail. En effet, si on veut bien considérer que les lettres [ qsdf jklm ] se trouvent sous les doigts placés en position de repos, sur la rangée médiane du clavier, mais que ces lettres ne sont pas nécessairement les plus fréquentes en français (ni en anglais ni en allemand non plus), on est là devant une belle aberration. Au cours des années 1930, le professeur August Dvorak avait repensé la distribution des caractères pour l'anglais en se fondant sur des critères linguistiques et non plus seulement mécaniques. Cela a donné le schéma ci-dessous :

\begin{tabular}{|c|c|c|c|c|c|c|c|c|c|c|c|c|c|c|c|}
\hline$\approx$ & $!$ & $\begin{array}{l}@ \\
2\end{array}$ & $\begin{array}{l}\# \\
3\end{array}$ & $\begin{array}{l}\$ \\
4\end{array}$ & $\begin{array}{l}\% \\
5\end{array}$ & & 6 & $\begin{array}{l}8 \\
7\end{array}$ & $\begin{array}{l}* \\
8 \\
8\end{array}$ & $\begin{array}{l}1 \\
9\end{array}$ & ) & & I & $\leftarrow$ & ackspace \\
\hline$\underset{\longrightarrow}{\leftarrow}$ Tab & ". & 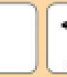 & & $s$ & $P$ & $Y$ & $\mathbf{F}$ & & $\mathbf{G}$ & C & R & L & $?$ & \pm & I \\
\hline$\theta_{\mathrm{Loc}}^{\mathrm{cap}}$ & $\begin{array}{l}\text { ps } \\
\text { ck }\end{array}$ & A & 0 & E & $\mathbf{U}$ & I & & D & $\mathrm{H}$ & $T$ & $\mathbf{N}$ & S & - & Enter & $\leftarrow$ \\
\hline 仓 $\mathrm{Shi}$ & & $\vdots$ & $\mathbf{Q}$ & $J$ & K & & $\mathbf{x}$ & B & M & W & & V & $\mathbf{Z}$ & 仓 Shift & \\
\hline trf & $=4 \mathrm{nx}$ & Alt & & & & & & & & & & AltGr & $f_{n x}$ & 厚 & Ctrl \\
\hline
\end{tabular}

51 Le confort d'écriture s'en ressent immédiatement: trajectoires significativement écourtées, meilleure distribution gauche/droite. À condition d'écrire uniquement en anglais, car le « dvorak anglophone » d'origine ne prévoit pas d'accents.

Faut-il préciser que les distributions de type «dvorak » sont largement tributaires des langues ou familles linguistiques qu'elles servent? Il est tout à fait illusoire de vouloir faire entrer toutes les langues sur une sorte de clavier « universel ", même si l'on s'en tenait aux langues à écriture latine. De même, une distribution purement alphabétique - qui aurait apparemment pour elle l'avantage de la simplicité - est en réalité difficilement utilisable. Un clavier universel devrait satisfaire plusieurs impératifs : répondre aux très fortes variations d'occurrences des lettres et des séquences de lettres dans les différentes langues, permettre d'accentuer tous les caractères accentués de toutes les langues...

En français, les travaux d'August Dvorak ont été repris par Claude Marsan qui a publié en 1976 une disposition destinée uniquement à la machine à écrire (le micro-ordinateur naissait à peine) et élaborée dans un but pédagogique puisque l'argument de Claude Marsan était de faciliter l'apprentissage de la lecture et de l'écriture par les enfants. Cette disposition n'a jamais été utilisée.

Les versions actuellement utilisées en français sont celles de Francis Leboutte ${ }^{28}$ et celle intitulée « bepo ». Dans les deux cas, les buts recherchés sont :

- un meilleur confort d'écriture avec des trajectoires plus courtes des doigts ;

- une meilleure symétrie des deux mains, avec une réelle alternance gauche/droite ;

- une diminution des mouvements de distorsion des doigts ;

- une accentuation aisée et correcte du français, et des langues des pays voisins ;

- une utilisation aisée autant pour les écrivants de texte que pour les programmeurs ;

- un temps d'apprentissage significativement plus court de la dactylographie.

Le français n'est pas la seule langue à connaître une effervescence "dvorakienne " destinée à optimiser le paramétrage du clavier en fonction de la langue écrite. Des créations sont en cours de montage pour l'allemand (Neo, Ristome, De-ergo), le turc, le finnois, le suédois et l'espagnol. 


\section{Adapter le clavier à la main}

56 L'adoption d'un paramétrage optimisé du clavier est lente, en dépit du réel confort procuré. En effet, tous ces développements sont profondément gênés dans leur diffusion par la forme matérielle des claviers qui s'ajoute à l'inertie des usages (Jacomy, 2001; Gardey, 1999). La dernière proposition, essentielle, serait en effet d'adapter enfin le clavier à la main, alors que jusqu'à présent, c'est la main qui s'est adaptée au clavier.

Le clavier classique, en tout cas le plus largement diffusé est un clavier dit «droit », mais ses touches sont disposées en quinconce, ce qui a pour conséquence notoire d'allonger significativement la distance à parcourir par les doigts. Pour un clavier de grande distribution, on a jusqu'à $19 \mathrm{~mm}$ entre le centre de deux touches voisines situées sur deux rangées différentes, centre où se place le doigt. De plus, cette disposition en quinconce complique sérieusement les parcours des doigts de la main gauche.

Bien que les claviers orthogonaux, c'est-à-dire dont les touches sont disposées à angle droit, soient depuis longtemps adoptés pour les caisses de supermarché, ou pour le téléphone, ils sont rarissimes pour les claviers d'écriture. Pour des touches de dimensions analogues, faites pour des doigts d'homme adulte, la distance entre deux centres de touches se trouve ramenée à 14 ou $15 \mathrm{~mm}$, soit presque diminuée d'un quart. Quelques fabricants développent ce type de produit, mais essentiellement pour des marchés de niche ${ }^{29}$.

Par ailleurs, on est frappé par le manque d'audace des fabricants (qui s'abritent derrière la norme ISO 9995) pour la forme même des claviers, notamment par la quasi-immuabilité de l'emplacement des touches de fonctions, des touches modificatrices, par le sousemploi des pouces, par l'incapacité à produire des claviers de différentes tailles, adaptées aux différentes tailles des mains (un peu comme les gants pour adultes dont la taille varie du $6 \frac{1}{2}$ au 11), adaptés à la longueur des doigts et à la palme (la largeur de la paume). Il y a certes des tentatives intéressantes avec les claviers de type " wave ${ }^{30}$ " qui sont faits pour les dactylographes, la plupart des fabricants faisant comme si tout le monde savait dactylographier... C'est un étrange malentendu, et pas le moindre résultat de l'invisibilité sociale du clavier. Il est difficile de décider si c'est l'incapacité à dactylographier qui entrave les efforts des fabricants, ou l'inertie de ces derniers qui gêne l'usage optimal.

Les recherches les plus fructueuses sur la forme du clavier et sur la distribution des touches (mais bizarrement assez peu sur la distribution des caractères) sont souvent inspirées par les recherches faites pour les handicapés manuels ou pour les handicapés neuro-moteurs (Al Faraj, 2010 ; Vella, 2008), mais aussi pour les joueurs avec des claviers plus proches de joysticks (qui se présentent sous forme de boule ou de manettes de jeu) que des claviers classiques.

\section{Conclusion}

61 Ce rapide état des lieux ne prétendait pas être exhaustif, d'autant que la réflexion sur le clavier conduit à s'interroger sur les usages sociaux, les pratiques professionnelles, les réponses des industriels à ce qu'ils estiment être la «demande » des utilisateurs et les effets de mode. 
62 pistes ci-dessus ne sont pas concurrentes, au contraire, elles s'étayent entre elles. Une formation généralisée à la dactylographie, sur des claviers optimisés par leur distribution de caractères et par leur forme matérielle, ainsi qu'une organisation du travail qui tienne compte de l'aspect répétitif des gestes d'écriture, constitueraient déjà une bonne base pour réduire de nombreux problèmes physiques provoqués par l'écriture. Car, si écrire est une activité physique à risque, souffrir en écrivant n'est pas une fatalité, d'autant que l'usage du clavier dans des conditions améliorées - et avec le potentiel bureautique désormais disponible - est beaucoup plus confortable que l'écriture au crayon.

\section{BIBLIOGRAPHIE}

AL FARAJ Khaldoun, Interaction gestuelle pour la saisie de textes en situation de handicap [en ligne], thèse de doctorat, Toulouse, IRIT, 2010. Disponible sur <http://thesesups.ups-tlse.fr/876/> [consulté le $1^{\text {er }}$ juin 2011].

AUSTER Paul, L'Histoire de ma machine à écrire, Paris, Actes Sud, 2003, 64 p.

CÉLINE Louis-Ferdinand, Mort à crédit [1936], Paris, Gallimard, coll. « Folio, n 1692 », 1990, 640 p.

FouRgous Jean-Michel, Réussir l'école numérique [en ligne], rapport de la mission parlementaire de Jean-Michel Fourgous, député des Yvelines sur la modernisation de l'école par le numérique, Paris, Documentation française, 2010. Disponible sur <http://www.educnet.education.fr/ actualites/archives/mars-2010/remise-du-rapport-fourgous> [consulté le $1^{\mathrm{er}}$ juin 2011].

GARDEY Delphine, « Mécaniser l'écriture et photographier la parole : utopies, monde du bureau et histoires de genre et de techniques » [en ligne], Annales, histoire, sciences sociales, Éditions de l'EHESS, no 3, 1999, p. 587-614. Disponible sur <http://www.persee.fr/web/revues/home/ prescript/article/ahess_0395-2649_1999_num_54_3_279766> [consulté le 1 ${ }^{\mathrm{er}}$ juin 2011].

Goody Jack, La Raison graphique : la domestication de la pensée sauvage, Paris, Éditions de Minuit, 1978, 272 p.

JАСому Bruno, L'âge du plip : chronique de l'innovation technique, Paris, Seuil, 2001, 241 p.

Jouvin Bruno, Le Siège de travail : choix et utilisation, Marseille, Éditions d'ergonomie, 2006, 92 p.

MAuss Marcel, «Les Techniques du corps », Journal de Psychologie, vol. XXXII, ns 3-4,

15 mars-15 avril 1936. Disponible sur <http://classiques.uqac.ca/classiques/mauss_marcel/ socio_et_anthropo/6_Techniques_corps/techniques_corps.pdf> [consulté le $1^{\text {er }}$ juin 2011]. NIEZBOROLA Michel et LAMY Anne, Travailler sans dérouiller : le bien-être au travail est-il encore possible?, Toulouse, éditions Milan, 2007, $141 \mathrm{p}$.

RAMAZINI Bernardino, Traité des maladies des artisans, trad. en 1778 par M. de Fourcroy (voir BNF cote NUMM 28586), édition Adolphe Delalhaie, Paris, 1855. Voir en particulier le chap. XLI « Maladies des imprimeurs » (p. 143) et le chap. XLII « Maladies des écrivains et des copistes » (p. 145). Disponible sur <http://gallica.bnf.fr/ark:/12148/bpt6k285867> [consulté le 1 er juin 2011]. 
Simondon Gilbert, Du mode d'existence des objets techniques, Paris, Aubier, 2001, 265 p.

TRÉGOUËT René, Rapport d'information $n^{\circ} 331$ fait au nom de la Commission des Finances, du contrôle budgétaire et des comptes économiques de la Nation, Paris, Sénat, 1998. Disponible sur < http://www.senat.fr/rap/r97-331-2/r97-331-21.html\#> [consulté le 1 ${ }^{\text {er }}$ juin 2011].

VANDERDORPE Christian, Du papyrus à l'hypertexte : essai sur les mutations du texte et de la lecture, Paris, La Découverte, 1999, 224 p.

VELLA Frédéric, Modèles psychophysiques d'atteintes de cibles pour les personnes souffrant de troubles neuromusculaires, thèse de doctorat, Toulouse, IRIT, 2008, 204 p. Disponible sur <http:// thesesups.ups-tlse.fr/560/1/Vella_Frederic.pdf> [consulté le $1^{\text {er }}$ juin 2011].

\section{NOTES}

1. Note nostalgique : l'intensité du «gling » déclenché par l'activation de ce qu'on a longtemps appelé « retour chariot » traduisait assez précisément l'humeur du dactylographe, gling gai et léger, gling rageur, selon les aléas de la vie.

2. Il y a une grande difficulté à estimer les pourcentages de frappe, car tout dépend de la nature et de la forme du texte utilisé pour l'expérience. Prendre un dictionnaire, par exemple, diminue la part tenue par la lettre « $\mathrm{s}$ » puisque les noms sont par principe mis au singulier et augmente considérablement la part de « $\mathrm{r}$ », car tous les verbes sont à l'infinitif. Le chiffre annoncé vaut pour des textes littéraires en français courant.

3. Voir à ce sujet l'exposition "Très toucher " au Palais de la Découverte, de novembre 2010 à mars 2011, puis de juin 2011 à février 2012 au Muséum de Nantes: <http://www.palaisdecouverte.fr/index.php?id=1978>.

4. Comptage empirique établi par des observations effectuées dans diverses entreprises, sur une trentaine de personnes en situation d'écriture professionnelle.

5. Le nombre moyen de caractères par mot est variable selon les langues.

6. Cette mention n'est indiquée que par quelques trop rares constructeurs de niches, et par aucun constructeur de grande diffusion.

7. Évaluation empirique, faite sur un clavier classique de grande distribution. Là aussi, le choix du clavier et le choix du corpus retenu peuvent infléchir le résultat, raison pour laquelle nous proposons plutôt de l'accepter pour le moment comme un ordre de grandeur à préciser.

8. Voir à ce sujet le site Dragon Naturally Speaking: <http://www.mysoft.fr/produit/ dragon_naturallyspeaking_11_reconnaissance_vocale.htm\#produit>.

9. «Amélie Nothomb en 2000 lettres », article de Christine Rousseau paru dans Le Monde des livres du $1^{\text {er }}$ octobre 2010.

10. «Article 8 - Le clavier doit être inclinable et dissocié de l'écran pour permettre au travailleur d'avoir une position confortable qui ne provoque pas de fatigue des avant-bras ou des mains. L'espace devant le clavier doit être suffisant pour permettre un appui pour les mains et les avantbras de l'utilisateur. Le clavier doit avoir une surface mate pour éviter les reflets. La disposition du clavier et les caractéristiques des touches doivent tendre à faciliter son utilisation. Les symboles des touches doivent être suffisamment contrastés et lisibles à partir de la position de travail normale. » Et dans sa nouvelle mouture du 7 mars 2008, le clavier est nommé « clavier de l'écran de visualisation ». Il perd ainsi toute personnalité propre et se retrouve assimilé à un rôle subalterne de «son excellence " l'écran. Cf. Journal officiel de la République française, Décrets $\mathrm{n}^{\circ}$ 91-451 du 14 mai 1991 et $n^{\circ}$ 2008-244 - R4542-7.

11. On remarquera la répartition des rôles entre les sexes, confirmée plus bas. 
12. Autre petit rappel nostalgique: Dactylos Rock chanté par les Chaussettes noires au début des années $1960:$ < http://www.dailymotion.com/video/xv3ds_les-chaussettes-noires-dactylorock_music>.

13. Un exemple littéraire dans Mort à crédit de Céline : Auguste, le père du narrateur prend des cours de dactylographie pour compléter sa compétence professionnelle. «On l'entendait au troisième comme il tapait sur sa machine, c'était un engin énorme, un clavier comme une usine... Quand il avait tapé longtemps ça lui tintait dans les oreilles le cliquetis des lettres, encore une partie de la nuit... Ça l'empêchait de s'endormir. [...] et puis, avec sa machine, c'était le comble des agaceries, jamais il pourrait s'y mettre !... Il passait devant des heures à essayer des « copies »... Il tapait dessus comme un sourd... Il crevait des pages entières... Ou bien il attaquait trop fort, ou bien pas assez, la petite sonnette arrêtait plus. De mon lit, moi j'étais tout près... Je le voyais bien s'escrimer... Comme il farfouillait dans ses touches, comme il s'empêtrait dans les tringles... [...] Monsieur Lempreinte, au bureau, s'acharnait toujours sur sa tronche, il le harcelait sans arrêt. [...] "Vous n'en finissez pas avec vos jambages! Ah! mon pauvre ami! regardez vos collègues! Ils ont terminé depuis longtemps! Vous êtes un calligraphe ! [...]" [...] dans une compagnie concurrente [...] Mais là aussi, faudrait qu'il tape... » (Céline, 1990, p. 288-289)

14. Passeport de compétences informatique européen.

15. "L'utilisation d'un clavier ou d'une souris peut être à l'origine de certaines douleurs ou lésions physiques graves. Lorsque vous travaillez sur ordinateur, comme pour de nombreuses autres activités, vous pouvez ressentir une gêne occasionnelle dans les mains, les bras, les épaules, le cou ou ailleurs. Toutefois, si des symptômes de gêne, de douleur, d'élancement, de picotement, d'engourdissement, de sensation de brûlure ou de courbature persistent, NE LES NÉGLIGEZ PAS. CONSULTEZ RAPIDEMENT UN MÉDECIN [en lettres capitales dans le texte], même s'ils apparaissent lorsque vous n'utilisez pas votre ordinateur. De tels signes peuvent en effet être liés à des lésions sévères, parfois définitives, ou à des troubles nerveux, musculaires, des tendons ou d'autres parties du corps. Ces troubles musculo-squelettiques incluent entre autres le syndrome du canal carpien, la tendinite et la ténosynovite. [...]» (Avertissement figurant au dos du clavier « Ergonomical » de Microsoft, acheté en 2007.)

16. L'accessoire le plus commun du clavier est la souris, outil de pointage, sorte de touche de fonction polyvalente, utilisée à la place des raccourcis-clavier qui, s'ils sont connus donnent lieu, le plus souvent, à des gesticulations effarantes. Ainsi le simple [CTRL + a] observé dans une entreprise toulousaine a fourni 9 séquences gestuelles différentes, dont un sujet particulièrement « débrouillard» qui à lui seul en utilisait quatre. On observe d'ailleurs avec amusement que les magasins d'informatique ont souvent un rayon de souris bien plus fourni que le rayon des claviers, que les vendeurs y sont plus prolixes et les emballages beaucoup plus séduisants.

17. Le terme "appuie-paume" lui est de loin préférable puisque c'est la paume qu'on peut éventuellement poser ; il commence à être adopté par les fabricants ; on peut aussi prendre appui sur les avant-bras.

18. Pour plus d'informations sur ces concours, voir Intersteno : <http://www.intersteno.fr/>. 19. Pour mémoire, un adulte lettré écrit à plus de 30 mots par minute avec un crayon.

20. Le vainqueur du concours 2009 à Pékin a écrit à la vitesse de 663,17 frappes par minute (soit l'équivalent de 120 mots en français par minute), résultat loin derrière le score établi en 2005 par Helena Matoušková avec 832,37 frappes par minute (soit l'équivalent de 151,34 mots français par minute) et un taux d'erreur de $0,08 \%$. Cf. Intersteno classement: <http://www.intersteno.it/ uploads/ClassificationsListVienna2005.pdf>.

21. La dénomination anglaise met en valeur la cause, le français la conséquence, mais il s'agit bien du même phénomène.

22. Qu'il s'agisse d'un logiciel marchand (par exemple, Ergosens : < http://www.zdnet.fr/ telecharger/logiciel/ergosense-11002959s.htm>), ou d'un logiciel gratuit (Workrave : <http:// www.workrave.org/>). 
23. Institut national de recherche et de sécurité pour la prévention des accidents du travail et des maladies professionnelles. Voir en particulier la brochure intitulée Le travail sur écran : <http:// www.inrs.fr/inrs-pub/inrs01.nsf/IntranetObject-accesParReference/INRS-FR/\$FILE/fset.html>.

24. Confort ne signifiant d'ailleurs pas amollissement.

25. Cf. Préventica : <http://www.preventica.com>.

26. Cf. Ergonoma : <http://www.ergonoma.com/index.php/fra/village-de-l-ergonomie-2.htm>.

27. Cf. Bureaux expo : <http://www.bureaux-expo.fr/>.

28. Dvorak-fr : version que j'utilise en formation car elle place les mains sur le bas sur le clavier (plus de $90 \%$ de la frappe d'un texte en français) et permet d'accentuer les langues d'Europe de l'Ouest. De plus, le parallélisme des mains est renforcé par la même fonction attribuée aux touches [Alt] et [Alt Gr], modification qui a en outre l'avantage de supprimer les frappes triples simultanées pour écrire les caractères.

29. Pour l'essentiel, il s'agit de fabricants nord-américains qui pratiquent des tarifs relativement élevés (entre 100 et $500 € \mathrm{HT}$ ). Pour les claviers spécialement conçus pour les handicapés manuels: Maltron; pour les «geeks»: Typematrix, Kynesys, Truly ergonomic; pour les « gamers » : Razer.

30. Comme le Logitech Wave : <http://www.logitech.com/fr-fr/68/5986>.

\section{RÉSUMÉS}

Écrire est ordinairement perçu comme une activité intellectuelle et uniquement sous cet angle. C'est aussi une activité physique, qui présente des spécificités et provoque des dommages corporels variés. Cette activité physique utilise de nos jours le clavier comme principal outil. Mais pour le malheur de ceux qui l'utilisent quotidiennement, c'est un outil invisible, déprécié par nos représentations sociales. Après avoir dressé un état des lieux, le présent article expose quelques pistes susceptibles de réconcilier la main, périphérique du cerveau humain, et le clavier, outil prolongeant la main. Ces pistes sont diverses et se confortent les unes les autres, que ce soit par la formation, par l'optimisation de la distribution des touches, par la création de nouvelles formes de clavier, par l'organisation du travail. Souffrir en écrivant n'est pas une fatalité.

Writing is essentially regarded as an exclusively intellectual activity. However, it is also a physical activity, with its own specific gestures which may generate various health problems. The main tool used in writing today is the keyboard. Unfortunately for those who use it on a daily basis, it is usually ignored and even poorly considered in the framework of current social representations. This article sets out to analyse this phenomenon and explore ways of reconciling the hand, as an extension of the human brain, with the keyboard, as an extension of the hand. Diverse solutions exist and complement each other, such as adequate training in typing, optimizing key distribution, creating new keyboard forms, organising work differently. Writing in sufferance is not fatality. 
INDEX

Mots-clés : clavier, dactylographie, frappe au clavier, Syndrome du canal carpien, TMS (troubles musculo-squelettiques)

Keywords : carpal tunnel syndrome, Keyboard, RSI (repetitive strain injury), typing

\section{AUTEUR}

MARTINE PINEAU

Professeure de dactylographie 